\title{
Simulation-Based Experimental Design and Statistical Modeling for Lead Time Quotation
}

\author{
Minqi Li ${ }^{\mathrm{a}}$, Feng Yang, ${ }^{\mathrm{a}, *}$ Hong Wan ${ }^{\mathrm{b}}$, John Fowler ${ }^{\mathrm{c}}$ \\ ${ }^{a}$ Industrial and Management Systems Engineering Department, West Virginia University, Morgantown, \\ WV 26505, USA \\ ${ }^{b}$ School of Industrial Engineering, Purdue University, West Lafayette, IN 47907, USA \\ ${ }^{c}$ School of Computing, Information, and Decision Systems Engineering, Arizona State University, Tempe, \\ AZ 85281, USA
}

\begin{abstract}
In a produce-to-order environment, it is of substantial interest to be able to quote a tight and reliable lead time for a new job (or order) upon its arrival to a manufacturing system. This work developed a simulation-based statistical approach to provide responsive and highquality prediction of a new job's flow time through the system, which renders the capability of accurately quoting lead times in real time. The approach integrates analytical queueing analysis, design of experiments, and statistical modeling to quantify the dependence of a new job's flow time distribution upon the shop status "seen" by that job. To the best of our knowledge, this is the first attempt to design simulation experiments in the space spanned by a complete set of shop status factors for the thorough investigation of the possible impacts those factors may have on the distribution of a new job's flow time. The method has been applied on a scaled-down semiconductor manufacturing system, and the quality of the quoted lead time has been evaluated based on a large and well-designed validation data set in terms of the six commonly-used performance criteria.
\end{abstract}

Keywords: simulation, lead time/due date quoting, design of experiments, regression, flow time estimation

\section{Introduction}

In a make-to-order production environment, the ability to quote a short and reliable lead time for a customer order is important to customer acquisition and retention [1]. In this paper, the lead time is defined as the time between the receiving of an order and the promised due date. For competitive and reliable quotation of lead time, it is essential to have a good prediction for the flow time of a new job (or order). However, at the time when the lead time needs to be quoted for a newly arrived order, it is difficult to predict its flow time, because the flow time of that job (i) depends on the current status of the shop floor

${ }^{*}$ Corresponding author. Tel.: +1 3042939477

Email address: Feng. Yang@mail.wvu.edu (Feng Yang )

Preprint submitted to Elsevier

June 13, 2014

(c) 2014. This manuscript version is made available under the Elsevier user license

http://www.elsevier.com/open-access/userlicense/1.0/ 
and (ii) is subject to the uncertainties inherent in the system (e.g., random processing time, machine failures, etc.).

A complete review of the lead time quotation literature is provided by Keskinocak and Tayur [1]. Here, attention is focused on the work most relevant to this paper; this stream of work considers a dynamic setting where customer orders arrive continually over time, and treats the lead time as an endogenous decision variable depending on shop status and subject to process uncertainties. The existing approaches in this stream can be roughly divided into two categories: analytical and empirical methods. The analytical methods mainly resorted to queueing theory to derive the flow time distributions of jobs [2-6]. Based on the derived flow time distribution, the lead time was set to optimize a certain objective or meet some criteria. Analytical models rely on restrictive assumptions such as Markovian property, and thus is far from adequate to provide an accurate flow time estimation for real systems that involve non-Markovian processing times, multiple workstations, machine failures, batching processings, etc.

The empirical methods, on the other hand, typically used simulation or real data to develop a mathematical model approximating the expected flow time as a function of job features and/or shop floor characteristics [7-10]. These empirical models in the literature range from linear regression models [11-15] to neural networks $[13,16,17]$. The advantage of an empirical model is that it can accommodate, at least in theory, any complexities present in the real system, since it is estimated from high-fidelity simulation or real data. However, several issues are yet to be addressed for the empirical modeling methods: (i) It is commonly recognized that the flow time of a new job depends on the shop status, but what set of state variables (e.g., the number of jobs at a workstation) constitutes a complete description of the shop floor? (ii) It typically takes a large number of state variables to completely describe the status of a real manufacturing system; how to identify those that play an important role in predicting a job's flow time, and how to obtain models accurately relating the flow time to the important predictors? (iii) How to characterize the flow time of a job with information beyond the first moment (expectation)? Higher moment information such as the flow time variance is particularly important to ensure the reliability of a quoted lead time. To generate high-quality models for lead time quotation, the questions above need to be adequately addressed, which motivated this work.

As an empirical approach, our method assumes the availability of a discrete-event simulation (DES) model representing the real system of interest, seeks to estimate from simulation data the statistical models that accurately quantify the dependence of a new job's flow time upon the shop status, and intends to provide a mechanism to quickly quote a tight lead time which is highly reliable. In light of the complexity of real manufacturing systems, a comprehensive framework was developed in this work, which includes preliminary analytical analysis, design of experiments (DOE), statistical modeling, and distribution fitting.

Specifically, our method differs from those in the literature in the following aspects. (i) 
First, it takes full advantage of a DES model, which provides and tracks a list of state variables completely describing the shop status at any time. (ii) Second, a DOE strategy was developed to specify the shop status (i.e., the complete set of state variables) that is encountered by a new job in simulation experiments. Given the typically large number of state variables, DOE is critical for correctly identifying those that have a big impact on a new job's flow time, as well as for achieving estimated statistical models of high quality [18]. In the literature, DOE has been completely ignored, at least partly due to the fact that it is challenging to design a shop status which involves large number of quantitative and qualitative state variables. (iii) Third, we model not only the mean but also the higher moments of flow time as a function of shop status; based on the moment estimates, a threeparameter Gamma distribution is fitted to describe the flow time of a new job, and used to quote its flow time. (iv) Fourth, preliminary analytical analysis is performed to facilitate the DOE and statistical modeling that involve large number of variables. Overall, to the best of our knowledge, this is the first attempt to develop a comprehensive statistical method which allows for a thorough investigation of the effects that various shop-status variables could have on a new job's flow time.

The developed method also renders some practical advantages over the existing work. First, it can be applied to any manufacturing system to obtain its important shop state variables and to perform statistical modeling for real-time flow time prediction. Second, it can be used to quote flow time for orders of varying size (i.e., multiple jobs). Third, the flow time quotation method can be easily adapted to predict the remaining flow time for jobs at any processing stage of the system. The ability to frequently update a job's progress as the system evolves serves two important purposes: Remedy actions can be taken and customers can be notified in advance if a job is likely to be late; If a high-priority order arrives, this information can be used to determine the sequence of the new order [2] while minimizing the negative impacts on the existing orders yet to be fulfilled.

The remainder of the paper is organized as follows. Section 2 provides an overview of the research problem addressed in this paper. Section 3.1 details the preliminary analytical analysis, and Section 3.3 discusses the statistical modeling issues for the flow time prediction. Section 3.4 is concerned with the design of experiments for estimating the regression models in Section 3.3. In Section 4, the proposed method is evaluated through a scaled-down semiconductor wafer fabrication system. A summary is given in Section 5 .

\section{Research Problem and Methodology Overview}

We are concerned with the lead time quotation for randomly-arriving customer orders to a manufacturing system, which may involve multiple workstations, machine failures, batch processing, re-entrant flows, etc. Although the proposed method can be extended to systems with job flows of multiple types, this paper primarily focuses on cases where all the jobs follow the same routing through the system. 


\subsection{Factors for Lead Time Quotation}

The key to quoting a good lead time lies in the ability to accurately predict flow time. The flow time of a newly arrived order may depend on the shop status upon arrival, the size of that new order, and the future order arrivals [11]. These three sources of factors are briefed respectively as follows. (i) The focus of this work is on investigating the dependence of flow time upon various shop characteristics, which will be detailed later in this section. (ii) As will be seen later (Section 3.3.3), a special modeling structure is developed in this paper to accommodate orders of varying sizes, that is, orders requiring different number of jobs. (iii) The arrivals of future orders may affect the flow time of the order being considered if the system involves re-entrant job flows (that is, jobs that need to be processed at the same station(s) for multiple times); with re-entrant flows, jobs from later orders may compete with earlier jobs for the same resources. In this work, the $1^{\text {st }}$ moment-characteristic of future orders is considered as a potential factor that may affect the quoted lead time. The characteristics of future order arrivals can be obtained from forecasting models, which is beyond the scope of this work.

We denote $\mathbf{x}_{O R I G}$ as the vector including all the three types of potential factors: (i) the shop status variables (SVs), (ii) the size of a new order, and (iii) the arrival rate of future orders. The objective of this work is to allow for real-time lead time quotation for a given $\mathbf{x}_{O R I G}$, which has been observed or recently forecasted.

The remainder of this section will discuss the SVs in details. A DES model of the system, which is assumed given in this work, provides and tracks a complete set of the SVs [19]. To clearly define these variables, we adopt the concepts of buffers [20]: A buffer is the virtual location of all jobs that are in the same station and the same production step.

We use the example system described in Appendix A for illustration. For that system, Figure 1 provides the sequence of stations that a job has to visit for processing. The sequence of buffer indexes, $\{B 1, B 2, \ldots, B 22\}$, shows that there are 22 processing steps required for each job. Some stations (i.e., Stations 1, 4, 5, 6 and 7) appear more than once in the job sequence, and thus need to be visited by a job for multiple times.

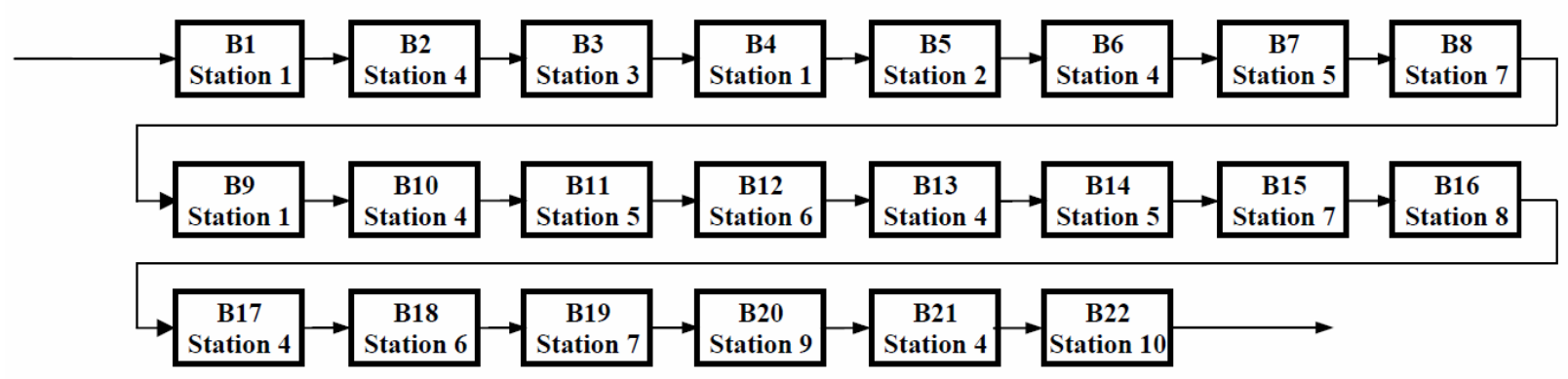

Figure 1: Job processing sequence.

For a manufacturing system, the set of SVs at a time instant typically includes several types of variables: 
SVs.A: The number of jobs at each buffer including those being processed and those waiting to be processed by the station associated with the corresponding buffer.

SVs.B: The status (busy or idle) of each server.

SVs.C: The elapsed processing time at each busy server, that is, the time that each currently busy server has been processing the job being served.

SVs.D: The status (up or down) of each server that is subject to random failures.

SVs.E: The elapsed down time for a currently down server.

SVs.F: The elapsed up time for a currently up server.

SVs.G: The batch size currently being processed at a batch processing server, if that server is busy at the moment.

The list above does not intend to exhaust all the SVs in DES models for manufacturing systems, but to provide the most commonly encountered and representative SVs. As will become clearer later, SVs not included in the list above can be statistically investigated in the same manner as those in the list.

\subsection{Overview of the Methodology}

Quantifying the dependence of flow time distribution upon $\mathbf{x}_{O R I G}$ (defined in Section 2.1) is difficult due to the typically large number of factors included in $\mathbf{x}_{O R I G}$, which poses a challenge to simulation-based DOE and statistical modeling.

To develop high-quality models relating a new order's flow time distribution to those important factors, we developed a comprehensive multi-stage framework, which is illustrated in Figure 2. Our method takes as inputs the simulation model for the target system and all the factors $\mathbf{x}_{O R I G}$ that may influence flow time. Due to the large size of $\mathbf{x}_{O R I G}$, a preliminary analytical analysis (Section 3.1) is first performed to derive a smaller set of relatively important variables $\mathbf{x}$, and $\mathbf{x}$, as opposed to $\mathbf{x}_{O R I G}$, will serve as the potential predictor variables of the flow time regression models. DOE strategies are developed to guide the simulation-based data collection for the estimation of the flow time vs. $\mathbf{x}$ relationships. From the simulation data, the regression models are estimated quantifying the functional dependence of flow time characteristics (i.e., the 1st moment, 2nd moment, and location parameter of the flow time) upon the predictor variables $\mathbf{x}$. These estimated models can be used for real-time lead time quotation without running any additional simulation, as will be articulated in Section 3.3.2. 


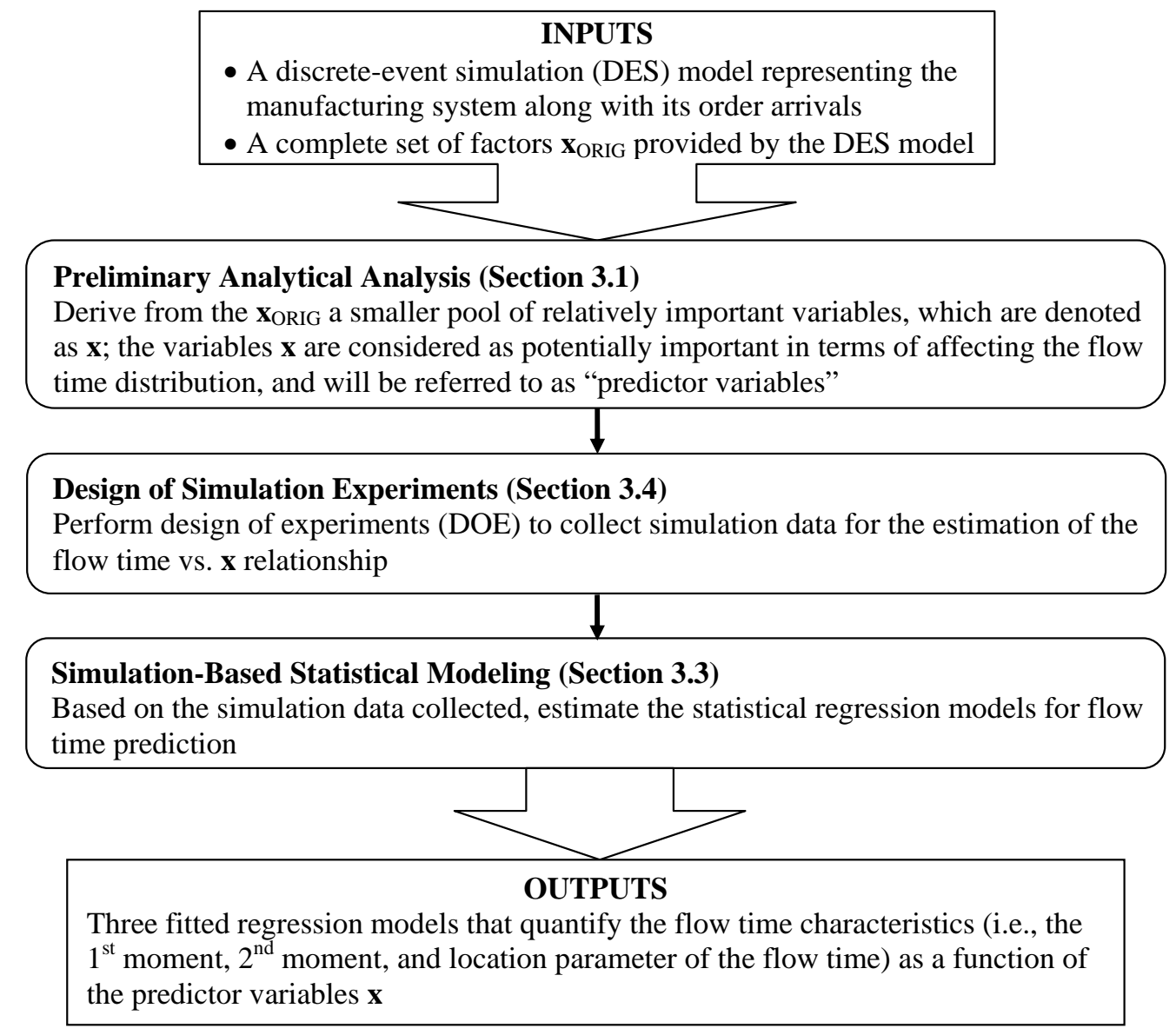

Figure 2: Overview of the methodology.

\section{Methodology}

\subsection{Preliminary Analytical Analysis}

The preliminary analytical analysis intends to obtain a smaller set of predictor variables $\mathbf{x}$ that are more likely to be important flow time predictors, by taking into account the relative importance of manufacturing workstations. Both queueing theory and empirical experience suggest that typically a system's performance is dominated by a small number of important workstations (IWs), which most constrain the job flows [21].

How to identify IWs? Highly-utilized stations are usually considered as IWs. For a given manufacturing system with certain job flows, the highly-utilized stations can be identified analytically. Specifically, suppose that the arrival rate of jobs is $\lambda$, then the utilization of each station $\left\{\rho_{j}(\lambda) ; j=1,2, \ldots\right\}$ can be calculated accurately (or even exactly) using existing queueing models in the literature. Such queueing models are given in [22-24], and are suitable for systems involving failures, re-entrant flows, batch processing, etc. Let $\rho_{\max }(\lambda)=\max _{j} \rho_{j}(\lambda)$, and $\rho_{j}(\lambda) / \rho_{\max }(\lambda)$ turns out to be a constant ratio. A rule of thumb adopted in this work is: If $\rho_{j}(\lambda) / \rho_{\max }(\lambda)>0.8$, then station $j$ is considered as highlyutilized station. In addition, the IWs may include any stations that the analyzer considers as important (say, based on experience with the real system). 
For instance, the analytical model developed by [22] has been applied on the example system (Appendix A) to identify the highly-utilized stations, which turn out to be Station 3, 4, and 8. These highly-utilized stations are considered as IWs, and are represented as shaded boxes in Figure 3. A buffer associated with IWs is considered as an important buffer (IB), and based on Figure 3, the IBs include: $\{B 2, B 3, B 6, B 10, B 13, B 16, B 17, B 21\}$. A server involved with IWs is referred to as an important server (IS).

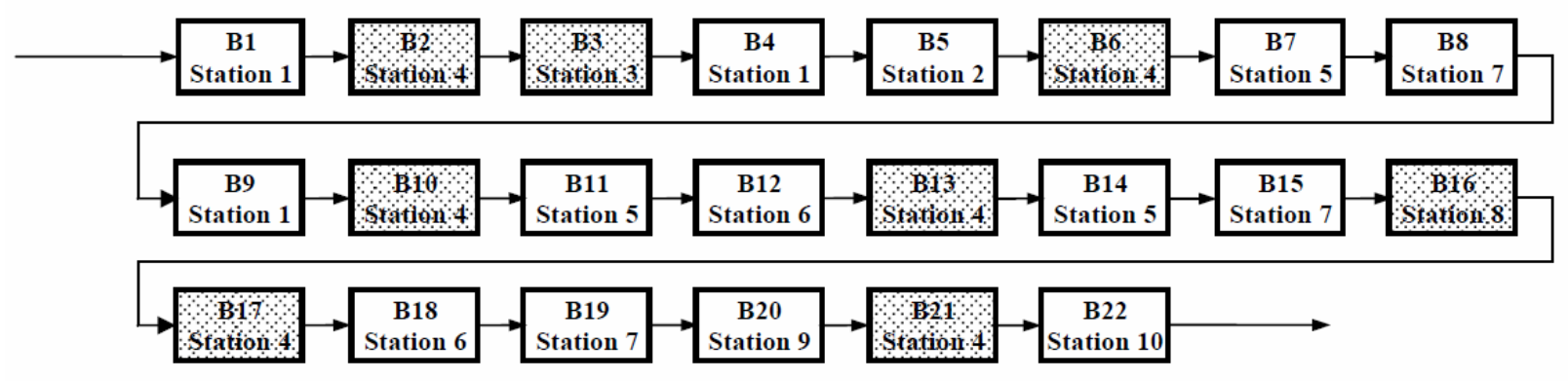

Figure 3: Important workstations.

With the identified IBs, the predictor variables $\mathbf{x}$ can be derived as follows from $\mathbf{x}_{O R I G}$. Recall from Section 2.1 that $\mathbf{x}_{O R I G}$ includes (i) the shop status variables SVs.A-G, (ii) the size of a newly arrived order, and (iii) the arrival rate of future orders, as also shown in Figure 4. Again, we use the example system for illustration.

First, instead of including the SVs.A, $\mathbf{x}$ includes a set of "stage WIP (work in process)" variables, which provide a good description of the total WIP as well as the WIP allocation throughout the system. These stage WIP variables are denoted as

$$
\mathbf{x}^{W I P}=\left(x_{1}^{W I P}, x_{2}^{W I P}, \ldots, x_{S}^{W I P}\right)
$$

with $S$ being the number of stages bounded by the IBs in a job sequence, and $x_{s}^{W I P}$ the number of jobs in the $s^{\text {th }}$ stage. How to specify stages bounded by IBs? Take the system shown in Figure 3 for an example: There are a total of 8 stages which include $B 1-B 2$, $B 3, B 4-B 6, \ldots, B 18-B 21$. The $1^{\text {st }}$ stage WIP $x_{1}^{W I P}$ counts the number of jobs yet to be processed by the station at the first IB $B 2$; the $s^{t h}(s=2,3, \ldots, S)$ stage WIP $x_{s}^{W I P}$ counts the number of jobs between the $(s-1)^{t h}$ and $s^{\text {th }}$ IB, including those being processed at the $(s-1)^{t h}$ IB and excluding those being processed at the $s^{t h}$ IB. The number of jobs that have been processed by the station at the last IB are not considered as part of the stage WIP variables. The variables $\mathbf{x}^{W I P}$ are defined as above because the IWs/IBs are where the queueing time and job congestion are most pronounced.

Second, we use $\mathbf{x}^{\text {non-WIP }}$ to represent all the other variables in $\mathbf{x}$ that are not stage WIP variables. The non-WIP variables $\mathbf{x}^{n o n-W I P}$ can be decomposed as

$$
\mathbf{x}^{n o n-W I P}=\left(\mathbf{x}^{B}, \mathbf{x}^{C}, \mathbf{x}^{D}, \mathbf{x}^{E}, \mathbf{x}^{F}, \mathbf{x}^{G}, x^{O}, x^{R}\right),
$$


where the component variables are defined as follows.

$\mathcal{I S}$ : The set of indexes of important servers (i.e., servers that belong to an IW)

$\mathcal{I S}_{u} \subset \mathcal{I S}:$ The set of indexes of important servers that are also subject to random failures

$\mathcal{I S}_{b} \subset \mathcal{I S}:$ The set of indexes of important servers that also involve batch processing

$\mathbf{x}^{B}=\left\{x_{i}^{B} ; i \in \mathcal{I} \mathcal{S}\right\}$ : the busy or idle status of important servers, which constitute a subset of SVs.B

$\mathbf{x}^{C}=\left\{x_{i}^{C} ; i \in \mathcal{I S}\right\}$ : the elapsed processing times at important busy servers, which constitute a subset of SVs.C

$\mathbf{x}^{D}=\left\{x_{i}^{D} ; i \in \mathcal{I S}_{u}\right\}$ : the status (up or down) of important servers subject to random failures, which constitute a subset of SVs.D

$\mathbf{x}^{E}=\left\{x_{i}^{E} ; i \in \mathcal{I S}_{u}\right\}:$ the elapsed down times for important down servers, which constitute a subset of SVs.E

$\mathbf{x}^{F}=\left\{x_{i}^{F} ; i \in \mathcal{I S}_{u}\right\}$ : the elapsed up time for each important up server, which constitute a subset of SVs.F

$\mathbf{x}^{G}=\left\{x_{i}^{G} ; i \in \mathcal{I S}_{b}\right\}$ : the batch sizes being handled by important busy servers involving batch processing, which constitute a subset of SVs.G

$x^{O}$ : the size of a newly arrived order

$x^{R}:$ the forecasted arrival rate of future orders

The relationships among the various factors are summarized in Figure 4. Derived from $\mathbf{x}_{\text {ORIG }}$, the variables in $\mathbf{x}$ are of relatively high importance and will be further employed to construct the flow time regression models. The predictor variables $\mathbf{x}$ are divided into two categories: WIP variables $\mathbf{x}^{\text {WIP }}$ as given in (1), and non-WIP variables $\mathbf{x}^{\text {non-WIP }}$ as given in (2). The WIP variables are singled out because they reflect the shop congestion, and are found to be the most important factors in flow time prediction [25].

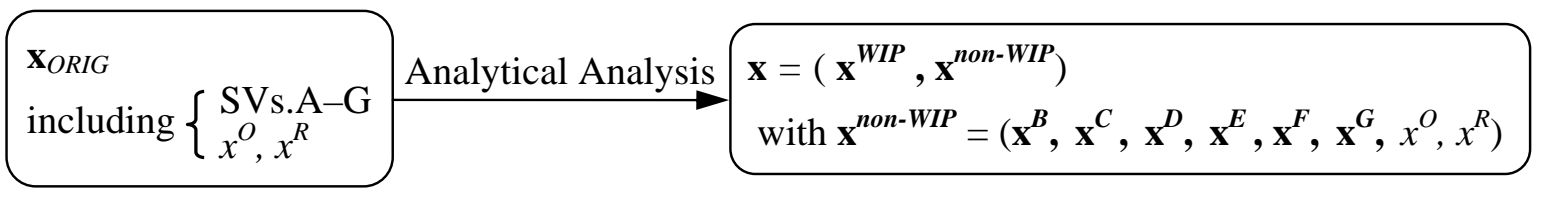

Figure 4: The relationships of the factor groups in this paper. 


\subsection{Simulation Experiments}

The example system (Appendix A) consists of 10 workstations, and involves machine failures, re-entrant flows, and batch processing. The customer orders come to the system according to a homogeneous compound Poisson process [26]; the size of each order follows the discrete uniform distribution, and is equally likely to be 1, 2, and 3. For specifics, please refer to Appendix A.

The simulation model for the system is coded in Microsoft Visual $\mathrm{C}++$. To develop regression models quantifying the dependence of flow time distribution upon the predictor variables $\mathbf{x}$, simulation experiments are carried out following the DOE method developed in Section 3.4. For each simulation run, $\mathbf{x}_{O R I G}$ is specified according to the DOE: A simulation run is initiated with a designed shop status and a newly-arrived order of a designed size at time 0 ; as the simulation proceeds, orders are fed into the system at the designed arrival rate; the simulation run is terminated once the new order generated at time 0 is completed, and its flow time is recorded.

\subsection{Simulation-Based Statistical Modeling for Flow-Time Prediction}

The flow time distribution is approximated by a three-parameter Gamma distribution whose probability density function is given as

$$
g(y ; \gamma(\mathbf{x}), \alpha(\mathbf{x}), \beta(\mathbf{x}))=\frac{1}{\Gamma(\alpha(\mathbf{x})) \beta(\mathbf{x})^{\alpha(\mathbf{x})}}[y-\gamma(\mathbf{x})]^{\alpha(\mathbf{x})-1} \exp \left\{-\frac{y-\gamma(\mathbf{x})}{\beta(\mathbf{x})}\right\}
$$

with $\alpha(\mathbf{x})$ being the shape parameter, $\beta(\mathbf{x})$ the scale parameter, and $\gamma(\mathbf{x})$ the location parameter. These parameters are all considered as dependent on the predictor variables $\mathbf{x}$. The distribution family (3) is selected because it is highly flexible [27], and is able to capture the shape of flow time distribution based on extensive empirical experience with manufacturing systems [28].

The modeling objective herein is to quantify the relationship between the predictor variables $\mathbf{x}$ and the flow time distribution, or equivalently the distribution parameters $\{\alpha(\mathbf{x}), \beta(\mathbf{x}), \gamma(\mathbf{x})\}$. Establishing such functional relationships relies on simulation data. As explained in Section 3.2, for each simulation run, the flow time of a newly arrived order is recorded with specified settings of $\mathbf{x}_{O R I G}$, from which the values of $\mathbf{x}$ can be easily obtained. The following notations are defined for convenience of discussion.

$K$ : the number of distinct sets of $\mathbf{x}$ values in the simulation experiments

$\mathbf{x}_{k}$ : the $k^{\text {th }}$ design point, i.e., the specified values for the $k^{\text {th }}$ distinct set of $\mathbf{x}$ values, with $k=1,2, \ldots, K$

$n\left(\mathbf{x}_{k}\right)$ : the number of simulation runs performed at the same setting of $\mathbf{x}_{k}$ 
$y_{j}\left(\mathbf{x}_{k}\right)$ : the flow time observation obtained from the $j^{\text {th }}$ simulation run under the setting of $\mathbf{x}_{k} ; j=1,2, \ldots, n\left(\mathbf{x}_{k}\right)$

$Y(\mathbf{x})$ : the random variable representing the flow time of a newly arrived order that "sees" the system settings as $\mathbf{x}$

Hence, the paired data collected from a total of $\sum_{k=1}^{K} n_{k}$ simulation runs can be represented as

$$
\left\{\left(\mathbf{x}_{k}, y_{j}\left(\mathbf{x}_{k}\right)\right) ; j=1,2, \ldots, n_{k} ; k=1,2, \ldots, K\right\}
$$

and used to estimate the flow time regression models.

The remainder of this subsection is organized as follows. Section 3.3.1 describes the simulation-based regression modeling that quantifies the dependence of the flow time upon $\mathbf{x}$. Section 3.3.2 articulates the use of the resulting regression models in real-time lead time quotation. Section 3.3.3 presents the specific functional form of the flow time regression models, which is a prerequisite for the regression analysis.

\subsubsection{Quantifying Flow Time Distribution}

In this work, the dependence of the distribution parameters $\{\alpha(\mathbf{x}), \beta(\mathbf{x}), \gamma(\mathbf{x})\}$ upon the predictor variables $\mathbf{x}$ is quantified indirectly through two steps. (i) First, based on the simulation data, three regression models are fitted relating $\mathbf{x}$ to the mean, variance and location parameter of the Gamma distribution, respectively. (ii) Second, once these regression models have been obtained, they can be used to estimate for any values of $\mathbf{x}$ the corresponding parameters $\{\alpha(\mathbf{x}), \beta(\mathbf{x}), \gamma(\mathbf{x})\}$, and thus provide a fitted Gamma distribution of the flow time.

The three regression models $\mathbf{F}(\mathbf{x}, \boldsymbol{\Theta})$ are written as:

$$
\begin{aligned}
\mathrm{E}[Y(\mathbf{x})] & =F_{1}\left(\mathbf{x}, \boldsymbol{\theta}_{1}\right) \\
\operatorname{Var}[Y(\mathbf{x})] & =F_{2}\left(\mathbf{x}, \boldsymbol{\theta}_{2}\right) \\
\gamma(\mathbf{x}) & =F_{3}\left(\mathbf{x}, \boldsymbol{\theta}_{3}\right)
\end{aligned}
$$

with the responses being the expected flow time, the variance of flow time, and the location parameter of the Gamma distribution, respectively. The unknown parameters involved are denoted as $\left\{\boldsymbol{\theta}_{i} ; i=1,2,3\right\}$.

To estimate the flow time models, the original simulation data (4) need to be transformed 
as follows:

$$
\begin{aligned}
\widehat{\mathrm{E}}\left[Y\left(\mathbf{x}_{k}\right)\right] & =\frac{1}{n_{k}} \sum_{j=1}^{n_{k}} y_{j}\left(\mathbf{x}_{k}\right) \\
\widehat{\operatorname{Var}}\left[Y\left(\mathbf{x}_{k}\right)\right] & =\frac{1}{n_{k}} \sum_{j=1}^{n_{k}}\left\{y_{j}\left(\mathbf{x}_{k}\right)-\widehat{\mathrm{E}}\left[Y\left(\mathbf{x}_{k}\right)\right]\right\}^{2} \\
\widehat{\gamma}\left(\mathbf{x}_{k}\right) & =2.2 y^{(1)}\left(\mathbf{x}_{k}\right)-1.2 y^{(2)}\left(\mathbf{x}_{k}\right)
\end{aligned}
$$

Clearly, (8) and (9) provide the mean and variance estimates at $\mathbf{x}_{k}$. The expression (10) is given by [29], and used to estimate the location parameter of the Gamma distribution at $\mathbf{x}_{k}$. In (10), $y^{(1)}\left(\mathbf{x}_{k}\right)$ and $y^{(2)}\left(\mathbf{x}_{k}\right)$ are the first two order statistics of $\left\{y_{1}\left(\mathbf{x}_{k}\right), y_{2}\left(\mathbf{x}_{k}\right), \ldots, y_{n_{k}}\left(\mathbf{x}_{k}\right)\right\}$.

Least square regression is performed to estimate Models (5), (6) and (7), from the data sets $\left\{\left(\mathbf{x}_{k}, \widehat{\mathrm{E}}\left[Y\left(\mathbf{x}_{k}\right)\right]\right) ; k=1,2, \ldots, K\right\},\left\{\left(\mathbf{x}_{k}, \widehat{\operatorname{Var}}\left[Y\left(\mathbf{x}_{k}\right)\right]\right) ; k=1,2, \ldots, K\right\}$ and $\left\{\left(\mathbf{x}_{k}, \widehat{\gamma}\left(\mathbf{x}_{k}\right)\right) ; k=\right.$ $1,2, \ldots, K\}$ respectively. Stepwise regression is used to select the parsimonious models. Once the fitted models for (5)-(7) have been obtained as $\widehat{\mathrm{E}}[Y(\mathbf{x})], \widehat{\operatorname{Var}}[Y(\mathbf{x})], \widehat{\gamma}(\mathbf{x})$, the distribution parameters can be estimated by recognizing:

$$
\begin{aligned}
\widehat{\alpha}(\mathbf{x}) & =\{\widehat{\mathrm{E}}[Y(\mathbf{x})]-\widehat{\gamma}(\mathbf{x})\}^{2} / \widehat{\operatorname{Var}}[Y(\mathbf{x})] \\
\widehat{\beta}(\mathbf{x}) & =\widehat{\operatorname{Var}}[Y(\mathbf{x})] /\{\widehat{\mathrm{E}}[Y(\mathbf{x})]-\widehat{\gamma}(\mathbf{x})\} \\
\widehat{\gamma}(\mathbf{x}) & =\widehat{\gamma}(\mathbf{x})
\end{aligned}
$$

\subsubsection{Lead Time Quotation in Practical Settings}

The three fitted regression models for (5)-(7) can be readily used for real-time lead time quotation without running any additional simulation. Suppose that the lead time is to be quoted for a newly arrived order with a desired service level of, say $95 \%[11,21]$. Upon the arrival of the new order, $\mathbf{x}_{O R I G}^{*}$ is observed and $\mathbf{x}^{*}$ derived. With the fitted regression models, $\widehat{\mathrm{E}}\left[Y\left(\mathbf{x}^{*}\right)\right], \widehat{\operatorname{Var}}\left[Y\left(\mathbf{x}^{*}\right)\right]$ and $\widehat{\gamma}\left(\mathbf{x}^{*}\right)$ can be estimated, and then lead to $\left\{\widehat{\alpha}\left(\mathbf{x}^{*}\right), \widehat{\beta}\left(\mathbf{x}^{*}\right), \widehat{\gamma}\left(\mathbf{x}^{*}\right)\right\}$, which fully specifies the flow time distribution for that new order. The lead time can be quoted as the corresponding percentile of the flow time distribution.

\subsubsection{Flow Time Regression Models}

Before any regression modeling can be performed, specific functional forms for Models (5)-(7) need to be assumed. Empirical efforts have been made to explore the adequacy of different model forms (e.g., polynomial, neural network, etc.) for simulation data. A quadratic form with dummy variables has been identified as the simplest and adequate one for the flow time models, which is evident from the regression results in Appendix Appendix B. The details are given below. 
Recall from Figure 4 that the predictor variables $\mathbf{x}$ can be decomposed as

$$
\mathbf{x}=\left(\mathbf{x}^{W I P}, \mathbf{x}^{n o n-W I P}\right)=\left(\mathbf{x}^{W I P}, \mathbf{x}^{B}, \mathbf{x}^{C}, \mathbf{x}^{D}, \mathbf{x}^{E}, \mathbf{x}^{F}, \mathbf{x}^{G}, x^{O}, x^{R}\right),
$$

with each group of factors defined in Section 3.1. The assumed functional form for Models (5)-(7) is represented as

$$
F(\mathbf{x}, \boldsymbol{\theta})=(I)+(I I)+(I I I)+(I V)+(V),
$$

and the functional components (I)-(V) are discussed as follows.

(I) The stage-WIP variables $\mathbf{x}^{\text {WIP }}$ are considered the most important predictors, and the functional terms involved in (I) are given as:

$$
(I)=\sum_{s=2}^{S} x_{s}^{W I P}+\sum_{s=2}^{S}\left(x_{s}^{W I P}\right)^{2}+\sum_{p=2}^{S-1} \sum_{q=p+1}^{S} x_{p}^{W I P} x_{q}^{W I P}+\left[x_{1 \sharp}^{W I P}+\left(x_{1 \sharp}^{W I P}\right)^{2}+\sum_{s=2}^{S} x_{1 \sharp}^{W I P} x_{s}^{W I P}\right] .
$$

Component (I) takes into account the main, quadratic, and interaction effects of $\mathbf{x}^{\text {WIP }}$. The variable $x_{1 \sharp}^{W I P}$ is singled out in (13) with

$$
x_{1 \sharp}^{W I P}=x_{1}^{W I P}+x^{O}
$$

being the sum of the $1^{\text {st }}$-stage WIP and the size of the new order, and (14) shows how the flow time models take into account the the order size $x^{O}$.

Note that in (13) and the discussions below for components (II)-(V), the unknown coefficients in front of the functional terms are omitted for clearance of presentation.

(II) This part concerns $\mathbf{x}^{B}$, the busy or idle status of important servers, $\mathbf{x}^{C}$, the elapsed processing times at important busy servers, and $\mathbf{x}^{G}$, the batch size being handled by important and busy servers involving batch processing. Since $\mathbf{x}^{C}$ and $\mathbf{x}^{G}$ come into play only for currently busy servers, we introduce a dummy vector $\mathbf{z}$, with each element being 0 or 1 corresponding to the "idle" or "busy" status of an important server, which is determined by the value of $\mathbf{x}^{B}$. The term (II) is expressed as:

$$
(I I)=\sum_{i \in \mathcal{I} \mathcal{S}} z_{i}\left[1+x_{i}^{C}+\left(x_{i}^{C}\right)^{2}\right]+\sum_{i \in \mathcal{I} \mathcal{S}_{b}} z_{i}\left[x_{i}^{G}+\left(x_{i}^{G}\right)^{2}\right]
$$

Here, the first and second order terms for $\mathbf{x}^{C}$ and $\mathbf{x}^{G}$ are considered.

(III) Term (III) concerns $\mathbf{x}^{D}$, the status (up or down) of the important servers subject to random failures, $\mathbf{x}^{E}$, the elapsed down times for important and currently down servers, and $\mathbf{x}^{F}$, the elapsed up times for important and currently up servers. Two 
dummy vectors are used for $\mathbf{x}^{D}: \mathbf{v}^{U P}$, with each element being 0 or 1 corresponding to the "down" or "up" status of an server; $\mathbf{v}^{D O W N}$, with each element being 0 or 1 corresponding to the "up" or "down" status of an server.

$$
(I I I)=\sum_{i \in \mathcal{I S}_{u}} v_{i}^{U P}\left[1+x_{i}^{F}+\left(x_{i}^{F}\right)^{2}\right]+\sum_{i \in \mathcal{I S}_{u}} v_{i}^{D O W N}\left[1+x_{i}^{E}+\left(x_{i}^{E}\right)^{2}\right]
$$

(IV) This term counts for the possible interaction effects between the status (up/elapsed up time, or down/elapsed down time) of an important unreliable server and the stage-WIP variable for the stage that immediately precedes the server.

$$
(I V)=\sum_{i \in \mathcal{I S}_{u}} v_{i}^{U P} x_{i}^{F} x_{s(i)}^{W I P}+\sum_{i \in \mathcal{I S}_{u}} v_{i}^{D O W N} x_{i}^{E} x_{s(i)}^{W I P}
$$

where $s(i)$ represents the index for the stage right before the important unreliable server $i$.

(V) Lastly, Term (V) is for $x^{R}$, the arrival rate of future orders, and is given as:

$$
(V)=x^{R}+\left(x^{R}\right)^{2}
$$

With the assumed functional form for Models (5)-(7), stepwise regression method is employed to search for the parsimonious models adequate to describe the data.

\subsection{Design of Experiments}

The DOE for fitting the flow time models (5)-(7) is challenging due to two major reasons. First, $\mathbf{x}$, the predictor variables for the flow time models, includes large number (21 in our example) of variables of different types: qualitative variables, and continuous/discrete quantitative variables. Designing experiments in such an input space is difficult [30, 31]. Second, a design in the space of $\mathbf{x}$ is not sufficient to specify the experimental condition of a simulation run which is represented as $\mathbf{x}_{O R I G}$. Hence, a design in the space of $\mathbf{x}$ has to be converted to one in the space of $\mathbf{x}_{O R I G}$.

In light of the difficulties above, a comprehensive design procedure is developed, and an overview is given in Figure 5. Through a series of steps, the procedure leads to a design including $K$ distinct design points in the space of $\mathbf{x}$ with $n_{k}(k=1,2, \ldots, K)$ replications each; and this design amounts to a total of $N=\sum_{k=1}^{K} n_{k}$ design points in the space of $\mathbf{x}_{\text {ORIG. }}$. The procedure input $K$ can be estimated as follows. In a practical context, the time available to develop the lead time quotation models (i.e., the three flow time models), which is typically abundant, can usually be estimated, since the simulation is performed "offline" prior to the need for decision making rises; the time it takes to complete one simulation run can also be estimated from preliminary simulation experiments. The ratio of the model 
development time and the simulation time per run provides an estimate of $N_{\max }$, the number of simulation runs allowed to develop the flow time models. Even though in the procedure, we allow the number of replications to vary at different design points, a rough estimate of $\bar{n}$, the average number of replications required, can be obtained from preliminary simulation experiments using the two-stage method in Section 3.4.6. Hence, $K$ can be set as $N_{\max } / \bar{n}$. The other inputs for the procedure (Figure 5) will be discussed as we proceed with this subsection.

Step 1-4 in Figure 5 are devoted to designing experiments in the space of $\mathbf{x}$, which can be divided as $\mathbf{x}=\left(\mathbf{x}^{W I P}, \mathbf{x}^{n o n-W I P}\right)$ (Figure 4$)$. The D-optimal criterion is adopted to find the $K$ design points for the $\mathbf{x}$ variables (Step 4) to optimize the quality of the estimated flow time models (5)-(7) following the common functional form (12). To facilitate the D-optimal design in the large space of $\mathbf{x}$, good candidate design points are first generated through Step 1-3, and then the $K$ optimal points are selected from the candidate pool by D-criterion (Step 4). In Step 5, the number of simulation replications is determined for each of the $K$ design points, and the design in the $\mathbf{x}$ space is converted to one in the $\mathbf{x}_{O R I G}$ space.

\subsubsection{Design for Total Congestion}

As shown in Step 1 of Figure 5, the procedure starts with selecting 4 levels for the total WIP $Q=\sum_{s=1}^{S} x_{s}^{W I P}$. Recall from (1) that $x_{s}^{W I P}$ represents a stage WIP variable in $\mathbf{x}^{W I P}$. A design for $Q$ is performed here out of the following considerations. First, $Q$ characterizes the overall congestion of a system, and has been demonstrated to be a good flow time indicator [11]. Even though in our work, the stage WIP variables $\mathbf{x}^{W I P}$, as opposed to the single total WIP, are used for flow time prediction, it is necessary for the final design to provide a good coverage in the range of total WIP. Second, designing experiments in the multi-dimensional integer space of $\mathbf{x}^{\text {WIP }}$ is difficult, with $x_{s}^{W I P}(s=1,2, \ldots, S)$ allowed to be a wide range of non-negative integers. As will be seen in Section 3.4.2, if a certain level of $Q$ is selected, a mixture design method can be easily adapted to address the design for $\mathbf{x}^{W I P}$.

Four evenly-spaced levels of $Q$ are recommended here over the range $\left[Q_{L}, Q_{U}\right]$ :

$$
Q_{L}, Q_{L}+\left(Q_{U}-Q_{L}\right) / 3, Q_{L}+2\left(Q_{U}-Q_{L}\right) / 3, Q_{U}
$$

The lower and upper bounds, $Q_{L}$ and $Q_{U}$, can be chosen based on the observed limits for real or simulation systems. Four levels are recommended for $Q$, because existing empirical results have shown that the expected flow time vs. $Q$ relationship is typically monotonicallyincreasing and convex and thus can be well captured by data collected at four distinct levels of $Q[11,32]$.

\subsubsection{Design for Stage WIP Variables $\mathbf{x}^{\text {WIP }}$}

Given a selected total WIP, say $Q^{*}$ (from Step 1, Figure 5), how to generate a set of candidate design points in the subspace of the stage WIP variables $\mathbf{x}^{\text {WIP }}$. Step 2 a seeks to 


\section{INPUTS}

- A discrete-event simulation (DES) model representing the manufacturing system of interest, along with the variable sets $\mathbf{x}_{\text {ORIG }}$ and $\mathbf{x}$ as defined in Figure 4

- Pre-specified number of distinct design points $K$ in the $\mathbf{x}$ space; the range of total WIP (work in process) denoted as $\left[Q_{L}, Q_{U}\right]$, the range of customer order size $\left[x_{L}^{O}, x_{U}^{O}\right]$

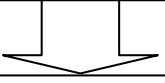

Step 1: Select 4 evenly-spaced levels for the total WIP (Section 3.4.1)

For each of the 4 levels of the total WIP, construct a D-Optimal design for the flow time model estimation following Step $2-4$ :

Step 2a: In the subspace spanned by WIP variables $\mathbf{x}^{\text {WIP }}$, generate a set of candidate design points (Section 3.4.2)

Step 2b: In the subspace spanned by nonWIP variables $\mathbf{x}^{\text {non-WIP }}$, generate a set of candidate design points (Section 3.4.3)

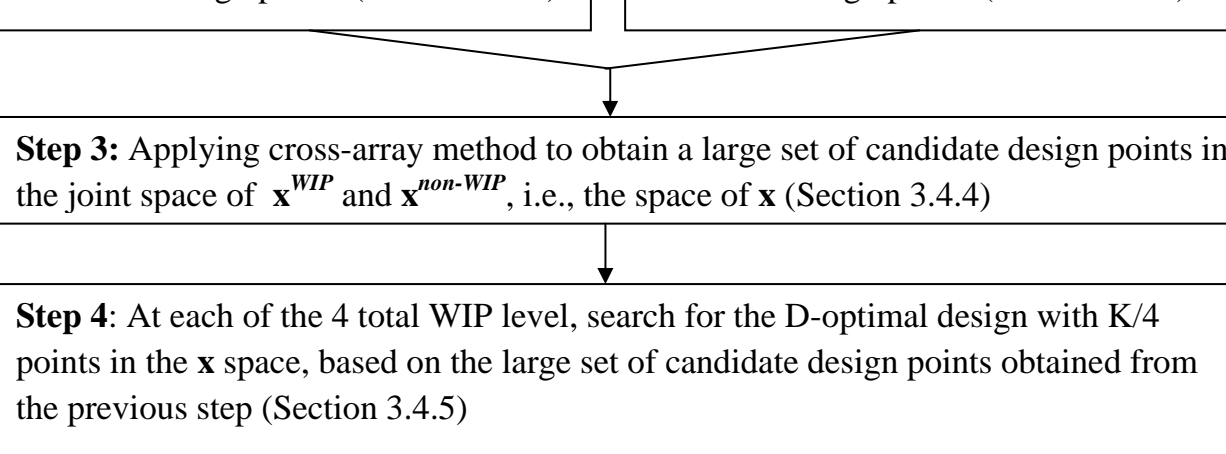

Combine the four D-Optimal designs (corresponding to four total WIP levels) as the final DOE in the $\mathbf{x}$ space, the space of predictor variables for flow time

\begin{tabular}{|c|c|}
\hline \multicolumn{2}{|c|}{$\begin{array}{l}\text { Step 5: For each of } K \text { design points in the } \mathbf{x} \text { space, determine the number of simulation } \\
\text { replications at that design point, and convert the design in the } \mathbf{x} \text { space to one in the } \\
\mathbf{x}_{\text {ORIG }} \text { space (Section 3.4.6) }\end{array}$} \\
\hline & $L$ \\
\hline $\begin{array}{l}\text { - The design in the } \mathbf{x} \text { space including } K \\
\text { number of simulation replications }\left\{n_{k}\right. \\
\text { - The converted design in the } \mathbf{x}_{\mathrm{ORIG}} \mathrm{Spa} \\
\text { corresponding to the design in the } \mathbf{x} \mathrm{s} F\end{array}$ & $\begin{array}{l}\text { UTS } \\
\text { ign points }\left\{\mathbf{x}_{k} ; k=1,2, \ldots, K\right\} ; \text { and the } \\
1,2, \ldots, K\} \text { assigned to each design point } \\
\text { with } \sum_{k=1}^{K} n_{k} \text { simulation runs) }\end{array}$ \\
\hline
\end{tabular}

Figure 5: The design of experiments procedure.

address this question as follows.

Let $\mathbf{p}=\mathbf{x}^{W I P} / Q^{*}$ be the vector of the proportions of the total WIP $Q^{*}$ assigned to each stage. With $Q^{*}$ given, we seek to obtain a design in the space of $\mathbf{x}^{\text {WIP }}$ by first generating a design in the space of $\mathbf{p}$; the latter can be easily converted to the former by noting that 
$\mathbf{x}^{W I P} \doteq \mathbf{p} \times Q^{*}$. The boundary constraints as

$$
\left\{\mathbf{p}_{L} \leq \mathbf{p} \leq \mathbf{p}_{U}\right\}
$$

may be imposed on the design space of $\mathbf{p}$. The lower and upper boundary vectors, $\mathbf{p}_{L}$ and $\mathbf{p}_{U}$, can be specified based on the system's historical data, or by running some preliminary simulation experiments.

To generate a design in $\mathbf{p}$ constrained by (20), we employ the uniform design algorithm for mixtures developed by [33]. This algorithm provides uniformly coverage [34] of the proportion space, and is able to deliver any specified number of distinct points. The number of points to generate for $\mathbf{p}$, denoted as $K^{W I P}$, can be selected in a somewhat arbitrary manner, since designing for $\mathbf{p}$ is only part of the effort to provide candidate design points in $\mathbf{x}$. We recommend setting $K^{W I P}$ as the number of central composite design [18] required for $S$ variables; since the flow time model involves full quadratic terms of the $S$ stage-WIP variables (13), which corresponds to the $S$ proportions.

Hence, Step 2a in Figure 5 leads to $K^{W I P}$ design points in the subspace of $\mathbf{x}^{\text {WIP }}$ constrained by a given total WIP.

\subsubsection{Design for non-WIP Variables $\mathbf{x}^{\text {non-WIP }}$}

Herein, we discuss Step 2b in Figure 5. To generate candidate design points in the subspace of the non-WIP variables $\mathbf{x}^{n o n-W I P}$, a mixed-level fractional factorial design [18] is used for $\mathbf{x}^{\text {non-WIP }}$ with several (typically two or three) levels selected for each variable.

For the variables $\mathbf{x}^{\text {non-WIP }}=\left(\mathbf{x}^{B}, \mathbf{x}^{C}, \mathbf{x}^{D}, \mathbf{x}^{E}, \mathbf{x}^{F}, \mathbf{x}^{G}, x^{O}, x^{R}\right)$, which are defined in Section 3.1, we select factor levels as follows. Each factor has a lower level (LL), high level (HL), and possibly a medium level (ML).

$x_{i}^{B}(i \in \mathcal{I S}):$ Two levels with LL and HL corresponding to the idle and busy status of server $i$.

$x_{i}^{C}(i \in \mathcal{I S})$ : Three levels with LL being 0 , HL the $95^{\text {th }}$ percentile of the distribution for server $i$ 's processing time, and ML the average of LL and HL.

$x_{i}^{D}\left(i \in \mathcal{I S}_{u}\right)$ : Two levels with LL and HL corresponding to the down and up status of server $i$.

$x_{i}^{E}\left(i \in \mathcal{I S}_{u}\right):$ Three levels with LL being 0 , HL the $95^{t h}$ percentile of the distribution for server $i$ 's repair time, and ML the average of LL and HL.

$x_{i}^{F}\left(i \in \mathcal{I S}_{u}\right):$ Three levels with LL being 0 , HL the $95^{\text {th }}$ percentile of the distribution for server $i$ 's time between failure, and ML the average of LL and HL.

$x_{i}^{G}\left(i \in \mathcal{I S}_{b}\right):$ Multiple levels with each one corresponding to a batch size allowed by server $i$. 
$x^{O}$ : Multiple (typically two) levels can be selected based on the distribution of the order size $x^{O}$. In the example system (Section 4), $x^{O}$ follows a discrete uniform distribution over the range $\left[x_{L}^{O}, x_{U}^{O}\right]=[1,3]$, and its LL, ML, and HL are set as minimum, average, and maximum of the distribution respectively.

$x^{R}$ : In this work, the future order's arrival rate $x^{R}$ takes three levels, which correspond to a steady-state system utilization of $75 \%, 85 \%$, and $95 \%$ respectively. With selected utilizations, the arrival rates can be determined using the queueing analytical analysis (Section 3.1), and for the example system, they turn out to be $0.387,0.455$, and 0.476 orders per hour.

To generate candidate design points in the $\mathbf{x}^{\text {non-WIP }}$ space, the mixed-level fractional factorial design method [18] is used to obtain a resolution-IV design for $\mathbf{x}^{\text {non-WIP }}$ with factor levels specified as above. A resolution-IV design [18] is chosen here which has clear main and interaction effects for the non-WIP variables. It is worth noting that some of the candidate points generated by the factorial design are infeasible. For instance, the variable setting of some design point involves an "up" and "busy" server $i$ which is also being repaired (with a positive value for $x_{i}^{D}$ ). However, we chose not to weed out these infeasible design points at this step, for reasons that will become clear in Section 3.4.5.

Thus, Step $2 \mathrm{~b}$ in Figure 5 ends up with, say $K^{\text {non-WIP }}$, candidate design points in the subspace of $\mathbf{x}^{\text {non-WIP}}$, which are determined by the resolution-IV factorial design.

\subsubsection{Candidate Design Points in the $\mathbf{x}$ Space}

In Step 3 of Figure 5, the cross array method [18] is used to generate candidate design points in the joint space of $\mathbf{x}=\left(\mathbf{x}^{W I P}, \mathbf{x}^{\text {non-WIP }}\right)$ based on (i) the $K^{W I P}$ points in the $\mathbf{x}^{\text {WIP }}$ subspace constrained by a given total WIP (Section 3.4.2), and (ii) the $K^{\text {non-WIP }}$ points in the $\mathbf{x}^{\text {non-WIP }}$ subspace (Section 3.4.3). The cross array obtained from these two subsets of points includes $K^{W I P} \times K^{\text {non-WIP }}$ design points in the space of $\mathbf{x}$. Again, among these points, some are infeasible and will be discussed and handled in Section 3.4.5.

The cross array design does not serve as the final DOE, and only provides a candidate pool for the D-optimal design in Section 3.4.5 for the following reasons. First, the cross array particularly aims at obtaining good estimates of the interaction effects between the WIP variables $\mathbf{x}^{W I P}$ and the non-WIP variables $\mathbf{x}^{\text {non-WIP }}[18]$, which is not the major concern in our modeling. Second, our DOE objective is to achieve flow time regression models of high quality, a popular measure of which is the D-criterion. However, searching for the D-optimal design directly in the feasible space of $\mathbf{x}$ is very challenging due to the large dimension of $\mathbf{x}$ and the different types of variables involved. The cross array provides a good candidate pool of design points, and substantially facilitates the D-optimal design. 


\subsubsection{D-Optimum Design}

For the cross array design obtained in Section 3.4.4 (Step 3, Figure 5), a large candidate design matrix $\widetilde{\mathbf{X}}_{C}$ can be constructed for the flow time models of the form (12). The matrix $\widetilde{\mathbf{X}}_{C}$ includes $K^{W I P} \times K^{\text {non-WIP }}$ rows, corresponding to $K^{W I P} \times K^{\text {non-WIP }}$ candidate design points in the cross array; each column represents a functional term in (12) evaluated across all the design points [35].

As mentioned earlier, the cross array includes infeasible design points caused by conflicting variable settings of the following two types. (i) First, conflicts may exist between WIP and non-WIP variables. By crossing the two subsets of points for $\mathbf{x}^{\text {WIP }}$ and $\mathbf{x}^{\text {non-WIP}}$, we may generate design points, for which a server is "busy" whereas the WIP level is 0 for the stage including that server. The rows of $\widetilde{\mathbf{X}}_{C}$ corresponding to such design points need to be deleted. (ii) Second, the settings of non-WIP variables may be conflicting, as explained in Section 3.4.3. However, the infeasible design points due to conflicting non-WIP variables turn out to be feasible rows in $\widetilde{\mathbf{X}}_{C}$. This is because each element in a row of $\widetilde{\mathbf{X}}_{C}$ corresponds to a specific functional term in the flow time model (12). In those functional terms, categorical variables are represented by dummy variables and coupled with their related and potentially conflicting variables as in (15) and (16), which eliminates the conflicts. Hence, only the rows corresponding to the infeasible points of type (i) are deleted from $\widetilde{\mathbf{X}}_{C}$, and we denote the cleaned-up design matrix as $\mathbf{X}_{C}$.

For each of the four selected total WIP levels (Section 3.4.1), there is a candidate design matrix $\mathbf{X}_{C}$, which serves as the candidate pool for the D-optimal design (Step 4, Figure 5). In this work, the Matlab function candexch was used to search for the D-Optimal design matrix $\mathbf{X}$ by solving the following optimization problem:

$$
\max _{\mathbf{X}}=\left|\mathbf{X}^{\prime} \mathbf{X}\right|
$$

Subject to: Each row of $\mathbf{X}$ is selected from the rows of $\mathbf{X}_{C}$

The matrix $\mathbf{X}$ is restricted to include $K / 4$ rows. Each row of $\mathbf{X}$ is selected from the rows included in the candidate matrix $\mathbf{X}_{C}$, and represents a design point in the $\mathbf{x}$ space. Hence, with four total WIP levels, $K$ design points in the space of $\mathbf{x}$ are generated by the procedure (Figure 5) and represented as

$$
\left\{\mathbf{x}_{k} ; k=1,2, \ldots, K\right\}
$$

with each element $\mathbf{x}_{k}$ being a design point.

\subsubsection{Simulation Replications and Design Conversion}

The primary goal of DOE is to achieve high-quality flow time models by providing a good design in the space of the important predictors $\mathbf{x}$. Through a series of steps (Step 1, 2a, 2b, 3 and 4 in Figure 5), a design including $K$ design points in the $\mathbf{x}$ space is obtained by the D-optimal criterion associated with the functional form (12). 
However, a design point in the $\mathbf{x}$ space, say $\mathbf{x}_{k}$, only specifies the settings of the stageWIP variables $\mathbf{x}^{W I P}$ and the important non-WIP variables $\mathbf{x}^{\text {non-WIP }}$, and thus is inadequate to specify all the experimental conditions (i.e., $\mathbf{x}_{O R I G}$ ) required by a simulation run. In addition, at a point $\mathbf{x}_{k}$, multiple replications are required to generate the estimates (8)-(10) needed for the flow time model fitting.

Hence, two remaining questions are yet to be answered: (i) How to determine $n_{k}$, the number of replications at $\mathbf{x}_{k}$ ? (ii) For a given $\mathbf{x}_{k}$, how to generate a specified number of design points in the space of $\mathbf{x}_{O R I G}$ (which corresponds to a certain number of replications at $\mathbf{x}_{k}$ )? Question (ii) is addressed in Appendix C. Here, we focus on the issue of determining $n_{k}$, which also involves calling the method in Appendix $\mathrm{C}$ as will be seen later.

The $n_{k}$ points in the $\mathbf{x}_{O R I G}$ space serve as the $n_{k}$ replications at $\mathbf{x}_{k}$, and are used to obtain the transformed data (8)-(9) for the fitting of the flow time models. Thus, $n_{k}$ is required to be sufficiently large to provide good estimates of (8)-(9). We chose to use the precision of the mean estimate (8) to drive the decision regarding $n_{k}$ through a two-stage framework [36].

Stage 1. A relatively small number of, say $n_{k}(0)$, simulation runs are performed for initial data collection. The value of $n_{k}(0)$ can be specified following the recommendations of [36], or based on preliminary simulation experiments. In our example (Section 4), we set $n_{k}(0)=50$ for $k=1,2, \ldots, K$.

For the given $n_{k}(0)$ and $\mathbf{x}_{k}$, the process in Appendix $\mathrm{C}$ is applied and the $n_{k}(0)$ design points in the space of $\mathbf{x}_{O R I G}$ are obtained. Following the design in the $\mathbf{x}_{O R I G}$ space, $n_{k}(0)$ simulation runs are carried out, and the following data are obtained: $\left\{\left(\mathbf{x}_{k}, y_{j}\left(\mathbf{x}_{k}\right) ; j=1,2, \ldots, n_{k}(0)\right\}\right.$.

Based on the initial data, $\widehat{\mathrm{E}}\left[Y\left(\mathbf{x}_{k}\right)\right]$ can be calculated following (8). The variance of $Y\left(\mathbf{x}_{k}\right)$ is estimated as

$$
\widehat{\operatorname{Var}}\left[Y\left(\mathbf{x}_{k}\right)\right]=\frac{1}{n_{k}(0)} \sum_{j=1}^{n_{k}(0)}\left\{y_{j}\left(\mathbf{x}_{k}\right)-\widehat{\mathrm{E}}\left[Y\left(\mathbf{x}_{k}\right)\right]\right\}^{2},
$$

and the standard error of $\widehat{\mathrm{E}}\left[Y\left(\mathbf{x}_{k}\right)\right]$ is estimated as $\mathrm{SE}\left\{\widehat{\mathrm{E}}\left[Y\left(\mathbf{x}_{k}\right)\right]\right\}=\sqrt{\widehat{\operatorname{Var}}\left[Y\left(\mathbf{x}_{k}\right)\right] / n_{k}}$. The following condition is used to determine the number of replications $n_{k}$.

$$
\frac{\mathrm{SE}\left\{\widehat{\mathrm{E}}\left[Y\left(\mathbf{x}_{k}\right)\right]\right\}}{\widehat{\mathrm{E}}\left[Y\left(\mathbf{x}_{k}\right)\right]}=\frac{\sqrt{\widehat{\operatorname{Var}}\left[Y\left(\mathbf{x}_{k}\right)\right] / n_{k}}}{\widehat{\mathrm{E}}\left[Y\left(\mathbf{x}_{k}\right)\right]} \leq p \%
$$

where $p \%$ is a user-specified precision level. In our empirical study, $p \%$ is set as $1.5 \%$. Hence,

$$
n_{k}=\left\lceil\frac{\widehat{\operatorname{Var}}\left[Y\left(\mathbf{x}_{k}\right)\right]}{\left(p \% \widehat{\mathrm{E}}\left[Y\left(\mathbf{x}_{k}\right)\right]\right)^{2}}\right\rceil
$$


Stage 2. A total of $n_{k}-n_{k}(0)$ simulation runs are performed at this stage. Given the replications $n_{k}-n_{k}(0)$ and $\mathbf{x}_{k}$, the process in Appendix $\mathrm{C}$ is applied again to generate $n_{k}-n_{k}(0)$ design points in the space of $\mathbf{x}_{O R I G}$. Accordingly, the additional simulation runs are carried out and the data obtained from both stages are denoted as $\left\{\left(\mathbf{x}_{k}, y_{j}\left(\mathbf{x}_{k}\right) ; j=1,2, \ldots, n_{k}\right\}\right.$.

The two-stage procedure is performed on each $\mathbf{x}$-space design point in $\left\{\mathbf{x}_{k} ; k=1,2, \ldots, K\right\}$, and leads to the final data set (4) for the model estimation.

\section{Empirical Results}

The developed method was applied on the scaled-down semiconductor fabrication system, which is described in Appendix A in detail. The complete set of $\mathbf{x}_{\text {ORIG }}$ for this system is given in Table B.2, Appendix B.

First, the analytical analysis (Section 3.1) was performed on the system, and the important stations and buffers were identified as in Figure 3. Consequently, $S=8$ stages, which are detailed in Section 3.1, are obtained with 8 stage-WIP variables: $\mathbf{x}^{W I P}=\left(x_{1}^{W I P}, x_{2}^{W I P}\right.$, $\left.\ldots, x_{8}^{W I P}\right)$. The non-WIP important variables $\mathbf{x}^{\text {non-WIP }}$ have also been identified and given Table B.3, Appendix B.

Once the predictor variables $\mathbf{x}=\left(\mathbf{x}^{W I P}, \mathbf{x}^{\text {non-WIP }}\right)$ have been identified, the DOE procedure (Figure 5) was applied with the following inputs: the DES model for the system being investigated; the number of design points $K=1000$, which is to be generated in the $\mathbf{x}$ space; the range of total WIP $\left[Q_{L}, Q_{U}\right]=[15,75]$, and the range of customer order size $\left[x_{L}^{O}, x_{U}^{O}\right]=[1,3]$.

Step 1 of the procedure (Figure 5) selects 4 evenly-spaced levels in the total WIP range: $\{15,35,55,75\}$. For each level of total WIP, 93, 312 candidate design points in the $\mathbf{x}$ space were resulted after the first three steps of the procedure, and 250 points were selected by removing the infeasible points and applying the D-criterion in Step 4. Hence, a total of $K=1000$ design points were generated in the $\mathbf{x}$ space. Through Step 5, the number of replications at each of the $K$ points was determined and the total number of simulation runs for this study turned out to be 169,061; the experimental settings (i.e., $\mathbf{x}_{\text {ORIG }}$ ) of these simulation runs were fully specified based on the $\mathbf{x}$-space design points. For this empirical case study, it took about 3 hours for a computer with Inter(R) Core(TM) i5 CPU and 8G RAM to complete all the simulation runs.

The simulation data obtained from the design above was used to fit the flow time regression models (5)-(7) following the method in Section 3.3.1. For the three fitted models, the adjusted $\mathrm{R}$ squares turned out to be $0.988,0.814$ and 0.973 respectively. The resulting flow time models are given in Appendix B as Model (B.1), (B.2) and (B.3). These models involve some first-order, second-order, and interaction terms of $\mathbf{x}^{W I P}$, some first-order terms of $\mathbf{x}^{n o n-W I P}$ and some interaction terms between $\mathbf{x}^{W I P}$ and $\mathbf{x}^{\text {non-WIP }}$. 
The three flow time models (B.1-B.3) given in Appendix B are ready to be used for realtime lead time quotation with desired reliability or service level, as articulated in Section 3.3.2. To evaluate the ability of these flow time models to quote lead time, a validation data set (VDS) was obtained which was designed to be different than the estimation data set (EDS), the data set used for model estimation. Specifically, to generate the VDS, the DOE procedure (Figure 5) was applied again with four changes: In Step 1, seven total WIP levels $\{10,20,30,40,50,60,70\}$ were selected which are all different from those in the EDS; the design method in Step 2a was applied on the WIP at each buffer (i.e., the SVs.A included in $\mathbf{x}_{O R I G}$ and defined in Section 2.1), as opposed to on the stage WIP variables for the EDS; the design method in Step $2 \mathrm{~b}$ was applied on $\mathbf{x}_{\text {ORIG }}$ excluding the SVs.A, as opposed to on the important non-WIP variables for the EDS; the procedure terminates after Step 3. The VDS includes $J=22400$ data pairs, and is denoted as

$$
\left\{\left(\mathbf{x}_{O R I G, j}^{*}, y_{j}^{*}\right) ; j=1,2, \ldots, J\right\}
$$

where $\mathbf{x}_{O R I G, j}^{*}$ represents a setting of $\mathbf{x}_{O R I G}$, and $y_{j}^{*}$ the observed flow time of the new job that "sees" the system condition as $\mathbf{x}_{O R I G, j}^{*}$.

Suppose that a service level of $\delta \%$ is required for the quoted lead time. Upon the arrival of a new job, the system setting is observed as, say $\mathbf{x}_{O R I G, j}^{*}$; then, $\mathbf{x}_{O R I G, j}^{*}$ is converted to the predictor variables $\mathbf{x}_{j}^{*}$, and fed to the flow time models (B.1)-(B.3) for $\left\{\widehat{\mathrm{E}}\left[Y\left(\mathbf{x}_{j}^{*}\right)\right], \widehat{\operatorname{Var}}\left[Y\left(\mathbf{x}_{j}^{*}\right)\right], \widehat{\gamma}\left(\mathbf{x}_{j}^{*}\right)\right\}$, from which the new job's flow time distribution is specified as Gamma with parameters $\left\{\widehat{\alpha}\left(\mathbf{x}_{j}^{*}\right), \widehat{\beta}\left(\mathbf{x}_{j}^{*}\right), \widehat{\gamma}\left(\mathbf{x}_{j}^{*}\right)\right\}$. Based on the fitted Gamma distribution, the $\delta^{t h}$ percentile of the distribution is quoted as the job's lead time and represented as $\ell_{j}$. The quoted lead time $\ell_{j}$ is evaluated by comparing it with the corresponding flow time $y_{j}^{*}$ $(j=1,2, \ldots, J)$ in the VDS via the following metrics:

- Achieved service level, which is calculated as: $\sum_{j=1}^{22400} I\left(y_{j}^{*} \leq \ell_{j}\right) / 22400$ with $I$ being the indicator function.

- Mean absolute percent error, which is calculated as: $(1 / 22400) \sum_{j=1}^{22400}\left|y_{j}^{*}-\ell_{j}\right| / y_{j}^{*}$.

- Mean earliness, which is calculated as: $(1 / 22400) \sum_{j=1}^{22400} \max \left(0, \ell_{j}-y_{j}^{*}\right)$.

- Mean tardiness, which is calculated as: $(1 / 22400) \sum_{j=1}^{22400} \max \left(0, y_{j}^{*}-\ell_{j}\right)$.

- Mean missed due date, which is calculated as: $(1 / 22400) \sum_{j=1}^{22400}\left|y_{j}^{*}-\ell_{j}\right|$.

- Mean of the lead time quoted, which is calculated as: $(1 / 22400) \sum_{j=1}^{22400} \ell_{j}$.

In the existing literature, Hopp and Sturgis' method [11], which will be referred to as HSM, was selected to be applied on the example system and compared with our method. HSM is the closest to our work in that it quotes the lead time of a desired service level based on the 
flow time distribution. It uses the total WIP as the sole factor to describe the dependence between the shop status and the distribution of new job's flow time.

Table 1 compares our method and HSM in terms of the six performance metrics with the target service level being $\delta \%=90 \%$ and $\delta \%=95 \%$ respectively. According to the row for "Achieved service level", our method is able to closely achieve the target service levels, whereas HSM overshoots the targets. The overly higher service levels given by HSM are provided by the longer HSM lead times, as evident by the row for "Mean of the lead time quoted". By quoting longer lead times, HSM performs slightly better in terms of "Mean tardiness", which is expected, but is substantially inferior in terms of "Mean earliness" and all the other performance measures in Table 1. Hence, overall our method is able to achieve the target service level with "tighter" lead times than those given by HSM.

Table 1: Comparison of our method and Hopp and Sturgis' method (HSM) in terms of the lead-time performance metrics

\begin{tabular}{lcccccc}
\hline & \multicolumn{2}{c}{$90 \%$ target service level } & & \multicolumn{2}{c}{$95 \%$ target service level } \\
\cline { 2 - 3 } \cline { 5 - 6 } Due date measures & Our method & HSM & & Our method & HSM \\
\hline \multirow{2}{*}{ Achieved service level } & 0.9027 & 0.9442 & & 0.9481 & 0.9697 \\
Mean absolute percent error & 0.1996 & 0.3625 & & 0.2626 & 0.4262 \\
Mean earliness (minutes) & 725.03 & 1355.90 & & 940.62 & 1610.87 \\
Mean tardiness (minutes) & 26.47 & 21.50 & & 12.86 & 9.8531 \\
Mean missed due date (minutes) & 751.49 & 1377.40 & & 953.48 & 1620.73 \\
Mean of the leadtime quoted (minutes) & 5234 & 5868 & & 5463 & 6135 \\
\hline
\end{tabular}

\section{Summary}

To quote a tight and reliable lead time for a new order upon its arrival, the key lies in the accurate prediction of that job's flow time through the manufacturing system of interest. A new job's flow time is difficult to predict, because it is not only subject to the randomness inherent in the production process, but may also depends on a typically large number of shop status variables (SVs) at the job's arrival time. To thoroughly investigate the impacts that the SVs may have on the random flow time, a comprehensive framework of methods is developed in this work. Assuming the availability of a discrete event simulation (DES) model for the system, a complete list of SVs fully capturing the shop status is obtained from the DES model, and investigated through a series of stages. First, a preliminary queueing analysis is performed to derive from the SVs a reduced number of important predictor variables for the flow time prediction. Second, a design of experiments (DOE) method is developed to specify the variable settings "seen" by new jobs in simulation experiments. The DOE aims at accurately estimating the functional dependence of the flow time distribution upon the predictor variables. Due to the large design space and the different variable types (i.e., qualitative and discrete/continuous quantitative variables), the DOE procedure includes a number of steps involving uniform mixture design, fractional factorial design, cross array, and D-optimal design. A good DOE method is a prerequisite for accurately quantifying the effects of predictor variables, and this work represents the first effort to design the shop 
status for simulation experiments. Third, from the simulation data collected following the DOE method, regression models are estimated quantifying the relationship between the characteristics (e.g., mean, variance, etc.) of the flow time distribution and the predictor variables.

These regression models are employed for real-time lead time quotation. Upon the arrival of a new job, the SVs are observed and the predictor variables obtained subsequently. Feeding the predictor variables to the regression models provide the estimated characteristics of the flow time distribution, which then lead to the fitted distribution that completely characterizes the job's random flow time. Based on the flow time distribution, the lead time of the job can be quoted with desired reliability or service level.

The developed method has been applied on a scaled-down semiconductor manufacturing system to obtain the regression models for lead time quotation. The ability of these models to quote lead time has been evaluated in terms of the six commonly-used performance metrics through a large well-designed validation data set.

\section{Acknowledgements}

This research was supported by National Science Foundation Grant CMMI-1068131.

\section{References}

[1] Keskinocak P, Tayur S. Due date management policies. In: SimchiLevi D, Wu SD, Shen ZJ, editors. Handbook of Quantitative Supply Chain Analysis: Modeling in the E-Business Era. Norwell, MA: Kluwer Academic Publishers; 2004, p. 485 - 547.

[2] Wein LM. Due-date setting and priority sequencing in a multiclass $\mathrm{m} / \mathrm{g} / 1$ queue. Manage Sci 1991;37(7):834-50.

[3] Duenyas I, Hopp WJ. Quoting customer lead times. Manage Sci 1995;41(1):43-57.

[4] Spearman ML, Zhang RQ. Optimal lead time policies. Manage Sci 1999;45(2):290-5.

[5] Savasaneril S, Griffin PM, Keskinocak P. Dynamic lead-time quotation for an $\mathrm{m} / \mathrm{m} / 1$ base-stock inventory queue. Oper Res 2010;58(2):383-95.

[6] Altendorfer K, Jodlbauer H. An analytical model for service level and tardiness in a single machine MTO production system. Int J Prod Res 2011;49(7):1827-50.

[7] Lawrence SR. Estimating flowtimes and setting due-dates in complex production systems. IIE Trans $1995 ; 27: 657-68$.

[8] Öztürk A. , Kayaligil S, Özdemirel NE. Manufacturing lead time estimation using data mining. Eur J Oper Res 2006;173:683-700.

[9] Pearn WL, Chung SH, Lai CM. Due-date assignment for wafer fabrication under demand variate environment. IEEE T Semiconduct M 2007;20(2):165-75.

[10] Baykasoğlu A, Göcken M, Unutmaz ZD. New approaches to due date assignment in job shops. Eur J Oper Res 2008;187:31-45.

[11] Hopp WJ, Sturgis LS. Quoting manufacturing due dates subject to a service level constraint. IIE Trans 2000;32:771-84.

[12] Vig MM, Dooley KJ. Dynamic rules for due date assignment. Int J Prod Res 1991;29:1361-77. 
[13] Hsu SY, Sha DY. Due date assignment using artificial neural networks under different shop floor control strategies. Int J Prod Res 2004;42(9):1727-45.

[14] Sabuncuoglu I, Comlekci A. Operation-based flowtime estimation in a dynamic job shop. Omega 2002;30:423-42.

[15] Sha DY, Storch RL, Liu CH. Development of a regression-based method with case-based tuning to solve the due date assignment problem. Int J Prod Res 2007;45(1):65-82.

[16] Philipoom PR, Rees LP, Wiegmann L. Using neural networks to determine internally-set due-date assignments for shop scheduling. Decision Sci 1994;25(5,6):825-51.

[17] Li SJ, Li Y, Liu Y, Xu YF. A GA-based NN approach for makespan estimation. Appl Math Comput 2007;185:1003-14.

[18] Wu CFJ, Hamada MS. Experiments: planning, analysis, and optimization. 2rd ed.; New York: Wiley; 2000.

[19] Law AM, Kelton WD. Simulation modeling and analysis. 3rd ed.; New York: McGraw-Hill; 2000.

[20] Riãno G. Transient behavior of stochastic networks: application to production planning with loaddependent lead times. Ph.D. thesis; Georgia Institute of Technology; 2003.

[21] Hopp WJ, Spearman ML. Factory physics: foundations of manufacturing management. Burr Ridge: Irwin/IMcGraw-Hill; 1996.

[22] Hopp WJ, Spearman ML, Chayet S, Donohue KL, Gel ES. Using an optimized queueing network model to support wafer fab design. IIE Trans 2002;34(2):119-30.

[23] Kumar S, Kumar PR. Queueing network models in the design and analysis of semiconductor wafer fabs. IEEE T Robotic Autom 2001;17(5):548-61.

[24] Meng G, Heragu SS. Batch size modeling in a multi-item, discrete manufacturing system via an open queuing network. IIE Trans 2004;36(8):743-53.

[25] Backus P, Janakiram M, Mowzoon S, Runger GC, Bhargava A. Factory cycle-time prediction with a data-mining approach. IEEE T Semiconduct M 2006;19(2):252-8.

[26] Gardiner CW. Handbook of stochastic methods. 2nd ed.; New York: Springer; 1983.

[27] Koutrouvelis IA, Canavos GC. Estimation in the three-parameter gamma distribution based on the empirical moment generating function. J Stat Comput Sim 1997;59(1):47-62.

[28] Yang F, Ankenman BE, Nelson BL. Estimating cycle time percentile curves for manufacturing systems via simulation. INFORMS J Comput 2008;20(4):628-43.

[29] Balakrishnan N, Wang J. Simple efficient estimation for the three-parameter gamma distribution. J Stat Plan Infer 2000;85:115-26.

[30] Wu CFJ, Ding Y. Construction of response surface designs for qualitative and quantitative factors. J Stat Plan Infer 1998;71:331-48.

[31] Draper NR, John JA. Response-surface designs for quantitative and qualitative variables. Technometrics 1988;30(4):423-8.

[32] Yang F, Ankenman BE, Nelson BL. Efficient generation of cycle time-throughput curves through simulation and metamodeling. Nav Res Log 2007;54:78-93.

[33] Fang KT, Yang ZH. On uniform design of experiments with restricted mixtures and generation of uniform distribution on some domains. Stat Probabil Lett 2000;46:113-20.

[34] Borkowski JJ, Piepel GF. Uniform designs for highly constrained mixture experiments. J Qual Technol 2009;41(1):35-47.

[35] Kutner MH, Nachtsheim CJ, Neter J. Applied linear regression models. 4th ed.; New York: McGrawHill/Irwin; 2004.

[36] Banks J, Carson JS, Nelson BL, Nicol D. Discrete-event system simulation. 5th ed.; Upper Saddle River, NJ: Prentice Hall; 2010.

[37] Kayton D, Teyner T, Schwartz C, Uzsoy R. Focusing maintenance improvement efforts in a wafer 
fabrication facility operating under theory of constraints. Prod Inv Manage J 1997;38(4):51-7. 


\section{Appendix A. Configuration of the Example System}

The example system investigated in this paper is a scaled-down semiconductor fabrication system developed by [37]. Customer orders arrive to the system according to a homogeneous compound Poisson process. The arrival rate of orders ranges within [0.194, 0.238] per hour, and the order size (i.e., number of jobs requested) follows the discrete uniform distribution with possible values being $\{1,2,3\}$.

Each job has to go through 22 processing steps, as shown in Figure A.1. The system includes 10 workstations, and involves the major features in real semiconductor fabrication system: re-entrant flows (Station 1, 4, 5, 6 and 7 are revisited by jobs), machine failures, and batch processing.

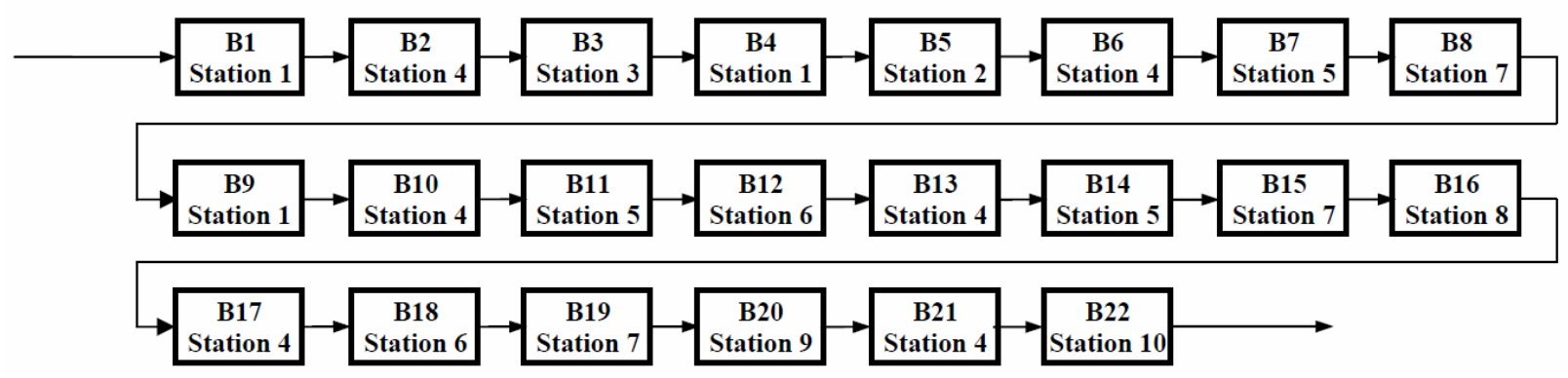

Figure A.1: Job processing sequence.

Table A.1 provides for each of the 10 workstations the following parameters: (i) number of machines available, (ii) whether or not the machines are subject to random failures, (iii) batch processing size, (iv) the mean of the processing time (Mean PT), and (v) the standard deviation of the processing time (Stdev PT). The processing time at each machine is log-normal.

Table A.1: Configuration of each workstation (Time units: minutes)

\begin{tabular}{ccccccccccc}
\hline Station Index\# & 1 & 2 & 3 & 4 & 5 & 6 & 7 & 8 & 9 & 10 \\
\hline \# of Machines & 1 & 1 & 1 & 2 & 1 & 1 & 1 & 1 & 1 & 1 \\
Batch Size (Min/Max) & $2 / 4$ & $2 / 4$ & 1 & 1 & 1 & 1 & 1 & 1 & 1 & 1 \\
Failure & No & No & Yes & No & No & No & Yes & No & No & No \\
Mean PT (minutes) & 80 & 220 & 80 & 40 & 25 & 22 & 40 & 100 & 50 & 50 \\
Stdev PT(minutes) & 7 & 16 & 7 & 4 & 2 & 2.4 & 4 & 12 & 4 & 5 \\
\hline
\end{tabular}

Machines at Station 3 and 7 are subject to random failures, with time to failure (TTF) and time to repair (TTR) both following the gamma distribution

$$
g(t, \alpha, \beta)=\frac{1}{\Gamma(\alpha) \beta^{\alpha}} t^{\alpha-1} \exp \left\{-\frac{t}{\beta}\right\} \quad t \geq 0
$$

At Station 3, the TTF follows the gamma with $(\alpha, \beta)=(3600,1)$, and the TTR has $(\alpha, \beta)=$ $(600,1.5)$; at Station 7 , the distribution parameters for TTF and TTR are $(\alpha, \beta)=(720,1)$ and $(\alpha, \beta)=(120,1.5)$ respectively. 
Station 1 and 2 involve batch processing. For both stations, the maximum and minimum batch sizes allowed are 4 and 2 respectively.

\section{Appendix B. Some Results of the Empirical Study}

Table B.2 lists all the variables in $\mathbf{x}_{O R I G}$, which are provided by the simulation model representing the example system. Applying the analytical model by [22] on the system, we

Table B.2: List of all the variables in $\mathbf{x}_{O R I G}$

\begin{tabular}{lc}
\hline Variables in $\mathbf{x}_{O R I G}$ & Number of Variables \\
\hline SVs.A: the number of jobs at each buffer & 22 \\
SVs.B: The status (busy or idle) of each server & 11 \\
SVs.C: the elapsed processing time at each busy server & 11 \\
SVs.D: The status (up or down) of each unreliable server & 2 \\
SVs.E: the elapsed down time for a currently down server & 2 \\
SVs.F: the elapsed up time for a currently up server & 2 \\
SVs.G: the batch size handled by a currently busy and batch-processing server & 2 \\
The size of a customer order & 1 \\
The forecasted arrival rate of future orders & 1 \\
\hline
\end{tabular}

identified 4 important workstations (IWs). Following the notations in Section 3.1, we have: $\mathcal{I S}=\{1,2,3,4\}, \mathcal{I S}_{u}=\{1\}$, and $\mathcal{I S}_{b}=\emptyset$. Based on the identified IWs, the relatively important predictor variables $\mathbf{x}$ are derived from $\mathbf{x}_{O R I G}$, and the variables included in $\mathbf{x}$ are listed in Table B.3.

Table B.3: Predictor variables for the flow-time modeling

\begin{tabular}{lcc}
\hline Variables in $\mathbf{x}$ & Notation & Number of Variables \\
\hline Stage WIP variables & $\mathbf{x}^{\text {WIP }}$ & 8 \\
SVs.B variables associated with IWs & $\mathbf{x}^{B}$ & 4 \\
SVs.C variables associated with IWs & $\mathbf{x}^{C}$ & 4 \\
SVs.D variables associated with IWs & $\mathbf{x}^{D}$ & 1 \\
SVs.E variables associated with IWs & $\mathbf{x}^{E}$ & 1 \\
SVs.F variables associated with IWs & $\mathbf{x}^{F}$ & 1 \\
SVs.G variables associated with IWs & $\mathbf{x}^{G}$ & 0 \\
The size of a customer order & $x^{O}$ & 1 \\
The forecasted arrival rate of future orders & $x^{R}$ & 1 \\
\hline
\end{tabular}

The simulation experiments were performed following the design of experiments resulted from applying the DOE method (Figure 5) on $\mathbf{x}$. From the simulation data collected, the three flow time models (5-7) were estimated as (B.1)-(B.3), and the analysis of variance (ANOVA) results are given in Table B.4-B.6 respectively. The ANOVA tables all suggest that the resulting regression models provide a good description of the data. As shown in (B.1)-(B.3), the WIP variables are the dominant factors for flow time prediction. The presence of the terms related to machine failures (e.g., $v_{1}^{U P}, v_{1}^{D O W N}, x_{1}^{E}$ and $x_{1}^{F}$ ) indicates that the failure behaviors of machines may also have a significant impact. The arrival rate of future orders $x^{R}$ appears in all the three prediction models for this system with re-entrant flows; and with the increase of $x^{R}$, that is, with more jobs coming to compete for limited resources, the flow time characteristics (i.e., the mean, variance, and minimum location) 
will increase as well. The responses of Model (B.1)-(B.3) are all positive given the feasible ranges of the predictable variables.

$$
\begin{aligned}
& \widehat{\mathrm{E}}[Y(\mathbf{x})]=365.94+154.98 x_{1 \sharp}^{W I P}+129.42 x_{2}^{W I P}+91.81 x_{3}^{W I P}+80.86 x_{4}^{W I P}+38.89 x_{5}^{W I P} \\
& +45.58 x_{6}^{W I P}+23.70 x_{8}^{W I P}+1.74 x_{1 \sharp}^{W I P} x_{3}^{W I P}+1.38 x_{1 \sharp}^{W I P} x_{5}^{W I P}+1.73 x_{3}^{W I P} x_{5}^{W I P} \\
& +1.27 x_{4}^{W I P} x_{5}^{W I P}+2.19 x_{4}^{W I P} x_{7}^{W I P}+1.90 x_{2}^{W I P} x_{3}^{W I P}+3.22 x_{5}^{W I P} x_{6}^{W I P}+1.33\left(x_{3}^{W I P}\right)^{2} \\
& +1.56\left(x_{4}^{W I P}\right)^{2}+2.44\left(x_{7}^{W I P}\right)^{2}+v_{1}^{W P}\left[-0.17 x_{1}^{F}+0.000057\left(x_{1}^{F}\right)^{2}\right]+16849.28\left(x^{R}\right)^{2}
\end{aligned}
$$

$$
\begin{aligned}
& \widehat{\operatorname{Var}}[Y(\mathbf{x})]=-100692.96+17013.36 x_{3}^{W I P}+8331.94 x_{4}^{W I P}+3411.24 x_{6}^{W I P}+10120.11 x_{7}^{W I P} \\
& +184.12 x_{1 \sharp}^{W I P} x_{4}^{W I P}+155.02 x_{1 \sharp}^{W I P} x_{5}^{W I P}+300.89 x_{1 \sharp}^{W I P} x_{8}^{W I P}-368.36 x_{3}^{W I P} x_{7}^{W I P} \\
& +171.11\left(x_{5}^{W I P}\right)^{2}+v_{1}^{U P}\left(-19.66 x_{1}^{F}\right)+v_{1}^{D O W N}\left(-71320.98+81.49 x_{1}^{E}\right)+594544.50 x^{R}
\end{aligned}
$$

$$
\begin{aligned}
& \widehat{\gamma}(\mathbf{x})=1004.68+83.06 x_{1 \sharp}^{W I P}+108.19 x_{2}^{W I P}+68.45 x_{3}^{W I P}+48.44 x_{4}^{W I P}+58.26 x_{5}^{W I P} \\
& +1.82 x_{1 \sharp}^{W I P} x_{3}^{W I P}+1.49 x_{1 \sharp}^{W I P} x_{4}^{W I P}+1.40 x_{1 \sharp}^{W I P} x_{7}^{W I P}+3.29 x_{3}^{W I P} x_{4}^{W I P}+1.33 x_{3}^{W I P} x_{7}^{W I P} \\
& +2.02 x_{4}^{W I P} x_{5}^{W I P}+1.56 x_{7}^{W I P} x_{8}^{W I P}+1.92 x_{1 \sharp}^{W I P} x_{2}^{W I P}+2.40 x_{5}^{W I P} x_{6}^{W I P}+0.99\left(x_{1 \sharp}^{W I P}\right)^{2} \\
& +2.32\left(x_{6}^{W I P}\right)^{2}+v_{1}^{U P}\left[-305.22+0.000022\left(x_{1}^{F}\right)^{2}\right]+v_{1}^{D O W N}\left(-0.322 x_{1}^{E}\right)+7828.65\left(x^{R}\right)^{2}
\end{aligned}
$$

Table B.4: The ANOVA results for the model of mean flow time (Model B.1).

\begin{tabular}{lrrrrr}
\hline Source & Degrees of Freedom & Sum of Squares & Mean Squares & $F$-Ratio & $P$-Value \\
\hline Model & 20 & $5.81 \times 10^{9}$ & $2.90 \times 10^{8}$ & 3953.97 & 0.000 \\
Residual & 979 & $7.19 \times 10^{7}$ & $7.35 \times 10^{4}$ & & \\
Total & 999 & $5.88 \times 10^{9}$ & & & \\
\hline
\end{tabular}

Table B.5: The ANOVA results for the model of flow time variance (Model B.2).

\begin{tabular}{lrrrrr}
\hline Source & Degrees of Freedom & Sum of Squares & Mean Squares & $F$-Ratio & $P$-Value \\
\hline Model & 13 & $1.88 \times 10^{13}$ & $1.45 \times 10^{12}$ & 330.98 & 0.000 \\
Residual & 986 & $4.31 \times 10^{12}$ & $4.37 \times 10^{9}$ & & \\
Total & 999 & $2.31 \times 10^{13}$ & & & \\
\hline
\end{tabular}

Table B.6: The ANOVA results for the model of Gamma location parameter (Model B.3).

\begin{tabular}{lrrrrr}
\hline Source & Degrees of Freedom & Sum of Squares & Mean Squares & $F$-Ratio & $P$-Value \\
\hline Model & 20 & $3.81 \times 10^{9}$ & $1.90 \times 10^{8}$ & 1779.49 & 0.000 \\
Residual & 979 & $1.05 \times 10^{8}$ & $1.07 \times 10^{5}$ & & \\
Total & 999 & $3.91 \times 10^{9}$ & & & \\
\hline
\end{tabular}




\section{Appendix C. Design Conversion}

The following task is addressed here: Given a design point $\mathbf{x}_{k}$ in the $\mathbf{x}$ space, how to generate a specified number, say $R$, points in the $\mathbf{x}_{O R I G}$ space? It is worth noting that answering this question is necessary, but the possibly many different ways to solve this problem are not expected to matter much to the flow time modeling results, since only the important variables in $\mathbf{x}_{k}$ are used to predict flow time. Hence, we simply present one venue to realize the task.

Recall the difference and relationship between $\mathbf{x}_{O R I G}$ and $\mathbf{x}$ (Figure 4), and two issues need to be addressed for the design conversion. First, the SVs.A variables (i.e., the number of jobs at each buffer) need to be specified for $\mathbf{x}_{O R I G}$, given the variables in $\mathbf{x}_{k}$ : the stage WIP variables $\mathbf{x}^{W I P}$ and the status (busy or idle) variables for important servers (ISs) $\mathbf{x}^{A}$. Specifically, for a stage $s$, the given stage WIP $x_{s}^{W I P}$ needs to be allocated to each buffer within that stage. The design method described in Section 3.4.2 is adopted again to perform the WIP allocation: Employ the uniform design for mixtures by [33] to obtain $R$ distinct mixtures, which leads to $R$ ways to allocate $x_{s}^{W I P}$ among the buffers. For Stage $s$, these $R$ allocation ways are denoted as $\left\{A_{s}^{(r)} ; r=1,2, \ldots, R\right\}$. The $R$ design points for all the SVs.A variables over the $S$ stages are represented as $\left\{\left(A_{1}^{(r)}, A_{2}^{(r)}, \ldots, A_{S}^{(r)}\right) ; r=1,2, \ldots, R\right\}$.

Second, the SVs.B-G variables associated with the unimportant stations are not included in $\mathbf{x}_{k}$, and need to be specified. Again, these variables are not expected to affect the flow time modeling results, and can be set in a somewhat arbitrary manner, as long as they are consistent with the SVs.A variables specified above. Below is what we did in the empirical case. The SVs.B variables are determined by the specified SVs.A already. For the unimportant SVs.C variables, if the server is busy according to the SVs.A, then set the elapsed processing time as the server's mean processing time. For the unimportant SVs.D-F variables, set the status of unreliable servers to be up and the elapsed up time the server's mean time between failures. For the unimportant SVs.G variables, if the batch processing server is busy according to the SVs.A, then set its batch size as the minimum. 


\section{Nomenclature}

IS the set of indexes of important servers

$\mathcal{I S}_{u} \quad$ the set of indexes of important servers that are also subject to random failures

$\mathcal{I S}_{b} \quad$ the set of indexes of important servers that also involve batch processing

$K \quad$ the number of distinct design points in the space of $\mathbf{x}$

$N \quad$ the number of design points in the space of $x_{\text {ORIG }}$

$S \quad$ the number of stages

SVs the shop status variables

$x_{\text {ORIG }}$ the vector including all the original variables that may impact the flow time

$\mathbf{x} \quad$ the vector including the predictor variables for flow time prediction servers 
Minqi Li is a PhD student in the Industrial and Management Systems Engineering Department at West Virginia University. His research interest lies in computer simulation of manufacturing systems, and applied statistics.

Feng Yang is an Associate Professor in the Industrial and Management Systems Engineering Department at West Virginia University. Her research interests include simulation and metamodeling, design of experiments, and applied statistics.

Hong Wan is an Associate Professor in the School of Industrial Engineering at Purdue University. She received her Ph.D. in Industrial Engineering and Management Sciences from Northwestern University. Her research interests include design and analysis of large-scale simulation experiments, simulation analysis of manufacturing and healthcare systems; quality management in supply chain, and quality control and applied statistics. She has taught a variety of courses and is a member of INFORMS and ASA. She currently serves as the associate editor of ACM Transactions on Modeling and Computer Simulation.

John W. Fowler is Chair and Professor of the W.P. Carey Supply Chain Management Department at Arizona State University. His research interests include modeling, analysis, and control of manufacturing and service systems. He is a Fellow of the Institute of Industrial Engineers and is the SCS representative on the Board of Directors of the Winter Simulation Conference. He is an Area Editor of Transactions of the Society for Computer Simulation International, an Associate Editor of IEEE Transactions on Semiconductor Manufacturing, and Editor of IIE Transactions on Healthcare Systems Engineering. 